\title{
Coronavirus disease 2019 (COVID-19): chest CT characteristics benefit to early disease recognition and patient classification-a single center experience
}

\author{
Hua Zhou ${ }^{1}$, Kaijin Xu ${ }^{2}$, Yihong Shen ${ }^{3}$, Qiang Fang ${ }^{4}$, Feng Chen ${ }^{1}$, Jifang Sheng ${ }^{2}$, Feng Zhao ${ }^{5}$ Haiyan Lou ${ }^{1}$ \\ ${ }^{1}$ Department of Radiology, The First Affiliated Hospital, Zhejiang University School of Medicine, Hangzhou, China; ${ }^{2}$ The State Key Laboratory \\ for Diagnosis and Treatment of Infectious Diseases, Collaborative Innovation Center for Diagnosis and Treatment of Infectious Diseases, The First \\ Affiliated Hospital, Zhejiang University School of Medicine, Hangzhou, China; ${ }^{3}$ Department of Respiration, ${ }^{4}$ Intensive Care Unit, ${ }^{5}$ Department of \\ Radiation Oncology, The First Affiliated Hospital, Zhejiang University School of Medicine, Hangzhou, China \\ Contributions: (I) Conception and design: H Zhou, F Chen; (II) Administrative support: K Xu, Y Shen, Q Fang, J Sheng, F Chen; (III) Provision \\ of study materials or patients: K Xu, Y Shen, Q Fang, J Sheng; (IV) Collection and assembly of data: K Xu, F Zhao, H Lou; (V) Data analysis and \\ interpretation: K Xu, F Zhao, H Lou, H Zhou; (VI) Manuscript writing: All authors; (VII) Final approval of manuscript: All authors. \\ Correspondence to: Feng Chen, MD, PhD. Department of Radiology, The First Affiliated Hospital, Zhejiang University School of Medicine, No. 79 \\ Qingchun Road, Hangzhou, China. Email: chenfenghz@zju.edu.cn.
}

Background: The current outbreak of coronavirus disease 2019 (COVID-19), epi-centered in Wuhan, Hubei Province of the China, has become a global health emergency. Several studies from China have recently provided the evidence of epidemiological, clinical, laboratory, and outcomes of COVID-19 patients. Investigation on the role of chest CT in patient screening and management course in a large cohort remains paucity.

Methods: This was a retrospective observational study based on the data collected between January 19 and 2020 to February 15, 2020. A clinic workflow using chest CT and RT-PCR assay to screen suspected patient was reviewed. Clinical data were evaluated and patients were classified to mild, common, severe and critical group. Chest CT characteristics of each patient were evaluated and a CT scoring system was applied to grade the lung involvement.

Results: Of 98 enrolled patients, 1, 29, 51 and 17 were clinically classified into mild, common, severe and critical group, respectively. Eighty-three patients $(84.7 \%)$ demonstrated ground-glass opacity (GGO), 76 patients $(77.5 \%)$ demonstrated consolidation and 18 patients $(18.4 \%)$ demonstrated crazy-paving pattern on chest CT. Based on the CT scoring, 2, 35, 55 and 6 patients were categorized to grade 0 , grade 1 , grade 2 and grade 3, respectively, which significantly consistent with clinical classification (kappa $=0.638$, $\mathrm{P}<0.05$ ). Twenty-nine patients admitted from fever clinic, with an average interval of 1.2 days (range, 0-4 days) between CT examination and onset of symptom. Three of these patients had negative initial RTPCR result while abnormalities displayed on the initial chest CT.

Conclusions: Peripheral lung distributed GGO and consolidation, without subpleural sparing, are the most common manifestations on chest CT of COVID-19. Abnormalities on chest CT can occur in an early stage of COVID-19, even when RT-PCR assay negative, which may help to early recognition and rapid diagnosis of this disease.

Keywords: Coronavirus disease 2019 (COVID-19); coronavirus; computed tomography; ground-glass opacity (GGO); pneumonia

Submitted Mar 02, 2020. Accepted for publication May 11, 2020.

doi: $10.21037 / \mathrm{atm}-20-2119$ a

View this article at: http://dx.doi.org/10.21037/atm-20-2119a 


\section{Introduction}

In late December 2019, a cluster of acute respiratory illness with unknown cause emerged in Wuhan, Hubei Province, China (1,2). Analysis of bronchoalveolar lavage fluid samples and electron microscopy revealed the etiology agent to be a novel coronavirus, which was temporarily named 2019 novel coronavirus (2019-nCoV) (3-6). As of late January 2020, tens of thousands of 2019-nCoV infected cases have been officially reported in China and sporadic cases also emerged in a growing number of international locations (7). On January 30, 2020, the novel coronavirus infection was declared as a global health emergency by the WHO and on February 11, 2020, the illness was renamed coronavirus disease 2019 (abbreviated "COVID-19").

Several studies from China have recently provided the first-hand evidence of epidemiological, clinical, laboratory and outcomes of 2019-nCoV infected patients $(6,8-10)$. Early disease recognition is crucial to speed up treatment and promptly isolate patient. Although CT examination has been widely applied to diagnose chest infection and a few recently published studies have described chest CT features of COVID-19 in limited cases (11-14), investigation on the role of $\mathrm{CT}$ in patient screening and management course in larger cohort remains paucity. Herein we report our experience in using chest $\mathrm{CT}$ examination to early detect COVID-19 pneumonia and categorize patients in a single center. We present the following article in accordance with the STROBE reporting checklist (available at http://dx.doi. org/10.21037/atm-20-2119a).

\section{Methods}

\section{Patients}

This was a retrospective observational study based on the data collected from the First Affiliated Hospital, Zhejiang University School of Medicine ( $1^{\text {st }}$ Hospital, ZJU), a major medical center located Hangzhou, Zhejiang Province, one of endemic areas of China. The hospital has been designated to be a specialized institution for receiving and treating COVID-19 patients by the Chinses government during the epidemic period. The approval for retrospective analysis of the patient data was obtained from ethics committee of $1^{\text {st }}$ Hospital, ZJU. And the patients' informed consent was waived for this retrospective case series. To avert potential breach of confidentiality, no link between the patients involved and the researchers was mad available.

From January 19, 2020, until February 15, 2020, a total of 100 consecutive COVID-19 infection patients were admitted to our hospital ( $1^{\text {st }}$ Hospital, ZJU). All these patients had reverse transcription-polymerase chain reaction (RT-PCR) assay of respiratory secretions or blood sample, and eventually obtained a positive result for COVID-19 nucleic acid. Among these 100 patients, 71 have been confirmed COVID-19 infection at other institutions before transferred to our hospital. The remaining 29 patients were admitted from our fever clinic. Of 71 transferred patients, 69 underwent at least one chest CT examination, and two patients were too ill to undergo CT examination.

Patients who introduced from fever clinic was selected followed a screening protocol drafted by our hospital (Figure 1). According to the "Guideline for Diagnosis and Treatment of 2019-nCoV Patients", which published by National Health Commission of China (Trial Version 5) (15), the criteria for suspected patients were as followed: (I) epidemiological history: travel/residence history in Wuhan, China or exposure to fever patients with respiratory symptoms from Wuhan within 14 days before the onset of illness; (II) clinical manifestations: fever, imaging characteristics of pneumonia, and/or normal or decreased leukocytes, and/or decreased lymphocyte count. All suspected patients were performed chest CT examination and RT-PCR test of respiratory secretions, which obtained by means of bronchoalveolar lavage, endotracheal aspirate, or nasopharyngeal swab. The initial chest CT examination and RT-PCR test for each patient were performed at the same day.

\section{Clinical characteristic and classification}

In addition to age and sex, clinical and epidemiological information included severity and time course of symptoms were obtained from the hospital's electronic medical records. According to the "Guideline for Diagnosis and Treatment of 2019-nCoV Patients", which published by National Health Commission of China (Trial Version 5), during management course, all 98 cases were classified into four groups: mild, common, severe and critical. The criteria were as follows: Mild group, cases with no symptom or light symptom present. Common group, cases with fever and/or respiratory symptoms. Severe group, cases meet any one of the following items (I) respiratory distress, $\mathrm{RP} \geq 30 / \mathrm{min}$; (II) $\mathrm{SpO}_{2} \leq 93 \%$; (III) $\mathrm{PaO}_{2} / \mathrm{FiO}_{2} \leq 300 \mathrm{mmHg}$. Critical group, cases meet any one of the following items: (I) respiratory failure required mechanical ventilation; (II) shock; (III) admission to intensive care unit (ICU) for other organ 


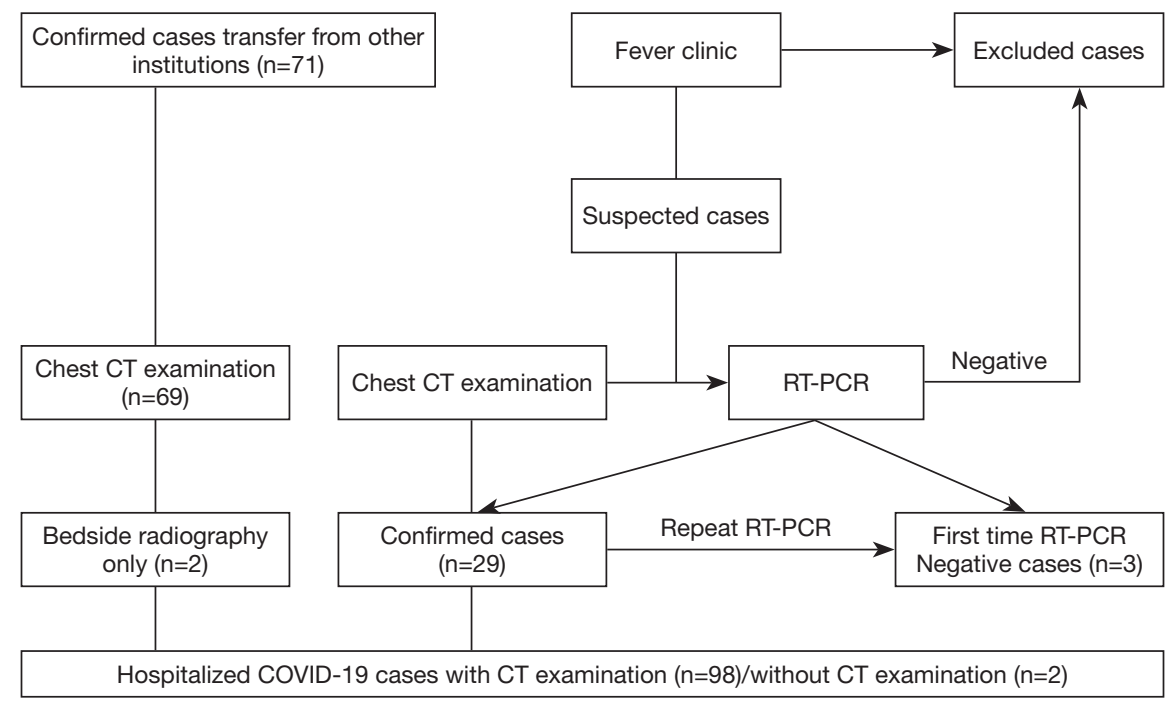

Figure 1 Diagram of a workflow for patient enrollment from fever clinic and other institutions.

failure.

\section{CT scan and imaging analysis}

CT scans were performed with a 128-slice CT scanner Revolution EVO (GE Medical Systems, Milwaukee, Wis) or a 64-slice CT scanner LightSpead VCT (GE Medical Systems, Milwaukee, Wis) with the following scanning parameters: $120 \mathrm{kVp}, 220-350 \mathrm{mAs}$. To minimize motion artifacts, patients were instructed on breath-holding; CT scan was performed during a single breath-hold. From the raw data, CT images were reconstructed as axial images with $0.625 / 1.25 \mathrm{~mm}$ slice thickness. $5 \mathrm{~mm}$ slice thickness images and three-dimensional (3D) and multiple planar reconstruction (MPR) images were generated at a workstation (Vitrea 2, Vital Images, Minnetonka, MN, USA).

All CT images were reviewed by two experienced radiologists ( $\mathrm{FZ}$ and $\mathrm{HL}$, with 8 and 21 years of experience in interpreting chest CT imaging, respectively), who were blind to the clinical information. The final interpretation was reached by consensus between the two radiologists, while a third investigator (FC, with 27 years of experience in interpreting chest CT imaging) took part in the arbitration when consensus could not be reached.

For each of the 98 patients, the interval from initial CT scan to onset of symptoms was assessed. The initial chest CT images were evaluated for the following characteristics: (I) presence of ground-glass opacity (GGO); (II) presence of consolidation; (III) presence of crazy-paving; (IV) presence of cavitation; (V) presence of mediastinal lymphadenopathy; (VI) presence of pleural effusion (16). The number of involved lung lobs and the lesion distribution were also noted. GGO was defined as hazy increased lung attenuation with preservation of bronchial and vascular margins, and consolidation was defined as opacification with obscuration of margins of vessels and airway walls. Crazy paving was defined as the appearance of GGO with superimposed interlobular septal thickening and intralobular septal thickening (17) (Figure 2).

Each of the lung lobes was assessed using a scoring system: $0=$ no involvement to a lobe $(0 \%), 1=$ minimal involvement to a lobe (1-25\%), $2=$ mild involvement to a lobe $(26-50 \%), 3=$ moderate involvement to a lobe (51$75 \%)$ and $4=$ severe involvement to a lobe $(76-100 \%)$. An overall score was obtained summing the cores of five lobe scores (range of possible scores, 0-20). And the severity of lung involved on CT scan was classified on a 4-point ordinal scale: grade 0 score of 0 (No abnormality present on $\mathrm{CT})$, grade one score of $1-5$, grade two score of $6-15$ and grade three score of 16-20 (11). If the patient has a series of CT scans, the most severe one during hospitalization was to be assessed using this scoring system.

\section{Statistical analysis}

The clinical characteristics and prevalence of chest CT findings were described as frequency rates and percentages. 

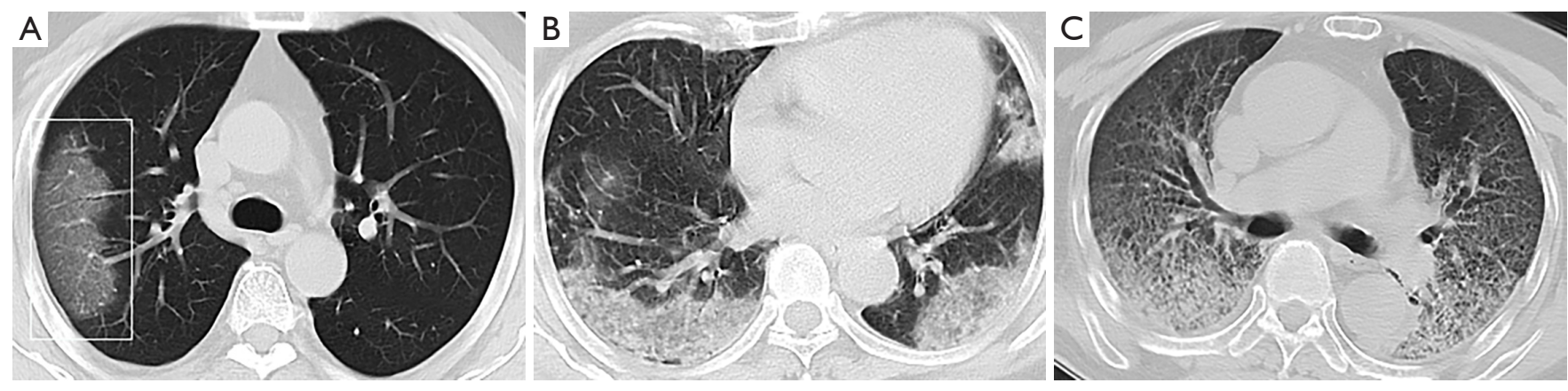

Figure 2 Imaging patterns of COVID-19. (A) Image in a 53-year-old man with history of recent travel to Wuhan who presented with fever, fatigue, and myalgia. Axial CT image shows GGO in the right upper lobe with pronounced peripheral distribution (box); (B) image in a 66-year-old woman who presented with dry cough and with history of contacting with confirmed COVID-19 patient. Axial CT image shows bilateral patch and confluent consolidative opacities in both lower lobes and left lingular segment; (C) image in a 91-year-old man who presented with fever and cough ultimately admitted to ICU. Axial CT shows a diffuse crazy-paving pattern in bilateral lung lobes.

Table 1 Summary of characteristics of 98 COVID-19 patients

\begin{tabular}{lc}
\hline Parameter & Value \\
\hline Sex & $58(59.2)$ \\
Men & $40(40.8)$ \\
Women & \\
Age (y) & 53.3 \\
Mean & $14-96$ \\
Range & \\
Signs and symptoms & $88(89.8)$ \\
Fever & $56(57.1)$ \\
Dry cough & $23(23.5)$ \\
Fatigue & $15(15.3)$ \\
Dyspnea & $11(11.2)$ \\
Myalgia & $7(7.1)$ \\
Headache & $5(5.1)$ \\
No obvious symptoms & $17(17.3)$ \\
Clinical classification & $29(29.5)$ \\
Mild & $51(52.0)$ \\
Common & \\
\hline Critical & \\
\hline
\end{tabular}

Data are numbers of patients, with percentages in parentheses.
Consistence between severity of lung involvement on chest $\mathrm{CT}$ and clinical classification was assessed using kappa test. Statistical analysis was performed using SPSS for Windows (version 20.0; SPSS Inc., Chicago, IL, USA).

\section{Results}

From January 19, 2020 to February 15, 2020, 100 consecutive COVID-19 infection patients were hospitalized in our hospital, and two cases were excluded for absence of chest CT scan (only bedside radiography provided). Therefore, there were 98 patients enrolled in the present study (58 men and 40 women), with a mean age of 53.3 years (range, 14-96 years). The most common symptoms at onset of illness were fever (88 of 98 patients) and dry cough (56 patients); less common symptoms were fatigue (23 patients), dyspnea (15 patients), myalgia (11 patients) and headache (7 patients). One, 29, 51 and 17 patients were clinically classified into mild, common, severe and critical group, respectively (Table 1).

Following the screening protocol for suspected COVID-19, 29 patients were admitted from our clinic. They underwent chest and RT-PCR test simultaneously on admission (Figure 1). Among these, three patients with history of epidemic exposure obtained negative results on initially RT-PCR assay while their chest CT examination demonstrated typical GGO or consolidation lesions. They 
were then isolated and had repeat RT-PCR. Two patients were positive at the second time of RT-PCR and another one remained negative until the sixth time of RT-PCR. Otherwise, three patients displayed normal images on initial chest CT, though ultimately confirmed COVID-19

Table 2 Chest CT findings in 98 COVID-19 patients

\begin{tabular}{|c|c|}
\hline Finding & Value \\
\hline GGO & $83(84.7)$ \\
\hline Consolidation & 76 (77.5) \\
\hline Crazy-paving & $18(18.4)$ \\
\hline \multicolumn{2}{|l|}{ No. of lobes affected } \\
\hline 0 & $2(2.0)$ \\
\hline 1 & $5(5.1)$ \\
\hline 2 & $12(12.2)$ \\
\hline 3 & $14(14.3)$ \\
\hline 4 & $31(31.6)$ \\
\hline 5 & $34(34.7)$ \\
\hline Peripheral distribution & $65(66.3)$ \\
\hline Bilateral distribution & $85(86.7)$ \\
\hline \multicolumn{2}{|l|}{ Total lung severity score } \\
\hline Mean & 8.6 \\
\hline Range & $0-20$ \\
\hline \multicolumn{2}{|l|}{ Other findings } \\
\hline Discrete pulmonary nodules & $0(0.0)$ \\
\hline Pleural effusion & $0(0.0)$ \\
\hline Lymphadenopathy & $0(0.0)$ \\
\hline
\end{tabular}

Data are numbers of patients, with percentages in parentheses. infection. One of them developed GGOs on the repeat CT image three days later; while the remaining two patients' chest CT images remained entirely normal during whole hospitalization.

For all enrolled 98 patients, the interval between the initial CT scan and the onset of symptom ranged from 0 to 9 days, with an average of 2.3 days. For 29 patients from fever clinic, that interval ranged from 0 to 4 days, with an average of 1.2 days. Excluding the two patients displayed normal chest CT image (0 lobe affected), 5 patients (5.1\%) had one affected lobe, 12 patients (12.2\%) had two affected lobes, 14 patients (14.3\%) had three affected lobes, 31 patients $(31.6 \%)$ had four affected lobes, and 34 patients (34.7\%) had disease affecting all five lobes (Table 2).

Out of the 98 patients, 83 cases $(84.7 \%)$ demonstrated GGO, 76 cases $(77.5 \%)$ demonstrated consolidation abnormality and 18 cases (18.4\%) demonstrated a crazypaving pattern (Figure 2). 65 cases $(66.3 \%)$ had a peripheral distribution and 85 cases (86.7\%) displayed bilateral involvement. No patient presented with cavitation in the lung, discrete pulmonary nodules, pleural effusion, or mediastinal lymphadenopathy (Table 2). The total lung severity score ranged from 0 to a maximum of 20, with a mean score of 8.6. Furthermore, 2, 35, 55 and 6 patients were radiologically categorized to grade 0 , grade 1 , grade 2 and grade 3, respectively. The radiological grading was significantly consistent with clinical classification (Table 3; Figures $3-5)(\mathrm{kappa}=0.638, \mathrm{P}<0.05)$.

\section{Discussion}

The current outbreak of COVID-19 (previously named 2019-nCoV), epi-centered in Wuhan, Hubei Province of the China, has become a global health emergency.

Table 3 Consistence between severity of lung involvement on chest CT series and clinical classification

\begin{tabular}{lccccc}
\hline \multirow{2}{*}{ Clinical classification } & \multicolumn{4}{c}{ Extent of severity of lung involvement on chest CT } \\
\cline { 2 - 6 } Mild & Grade 0 & Grade 1 & Grade 2 & Grade 3 & 0 \\
Common & 1 & 0 & 0 & 1 & 0 \\
Severe & 1 & 27 & 43 & 0 & 51 \\
Critical & 0 & 8 & 11 & 6 & 17 \\
Total & 0 & 0 & 55 & 6 & 98 \\
\hline
\end{tabular}

Data are numbers of patients, with percentages in parentheses. Significant consistence existed between severity of lung involvement on chest CT scan and clinical classification (kappa $=0.638, \mathrm{P}<0.05)$. 

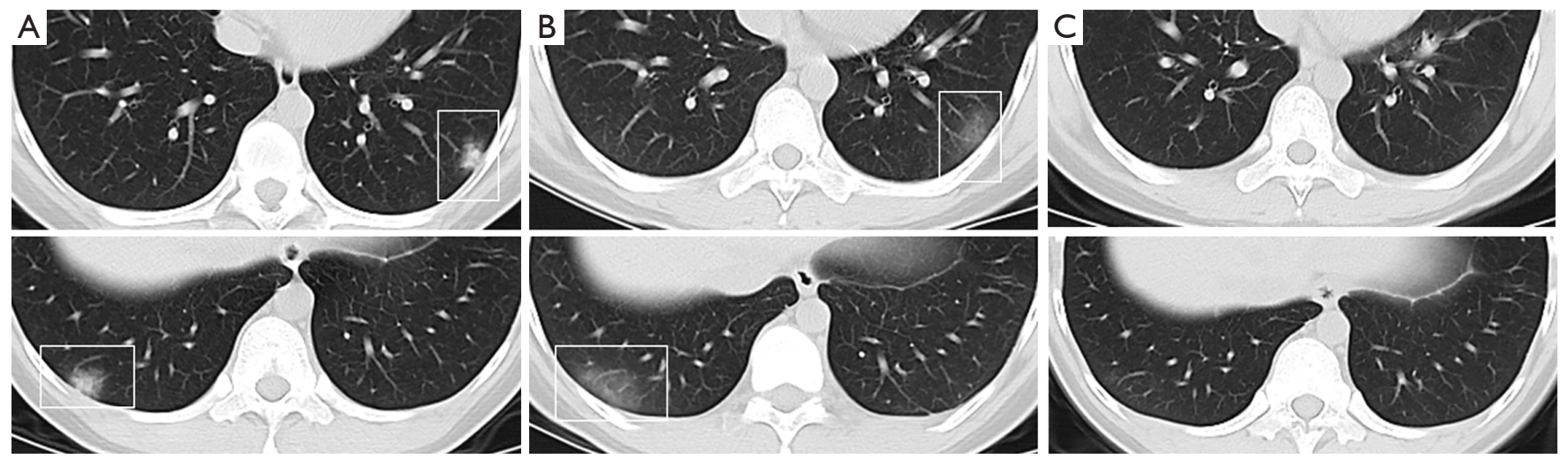

Figure 3 A 24-year-old woman with history contacting colleagues from Wuhan presented with fever for one day. (A) The initial CT scan performed on the second day after onset of symptom depicts subtle subpleural GGO and consolidation on bilateral lower lobes (box); (B) axial image obtained 10 days later shows the lesions have become less dense but larger (box); (C) twenty days later, lesions continued to resolve and almost disappeared. This case was clinically classified to common group, which consistent with radiological grade 1.
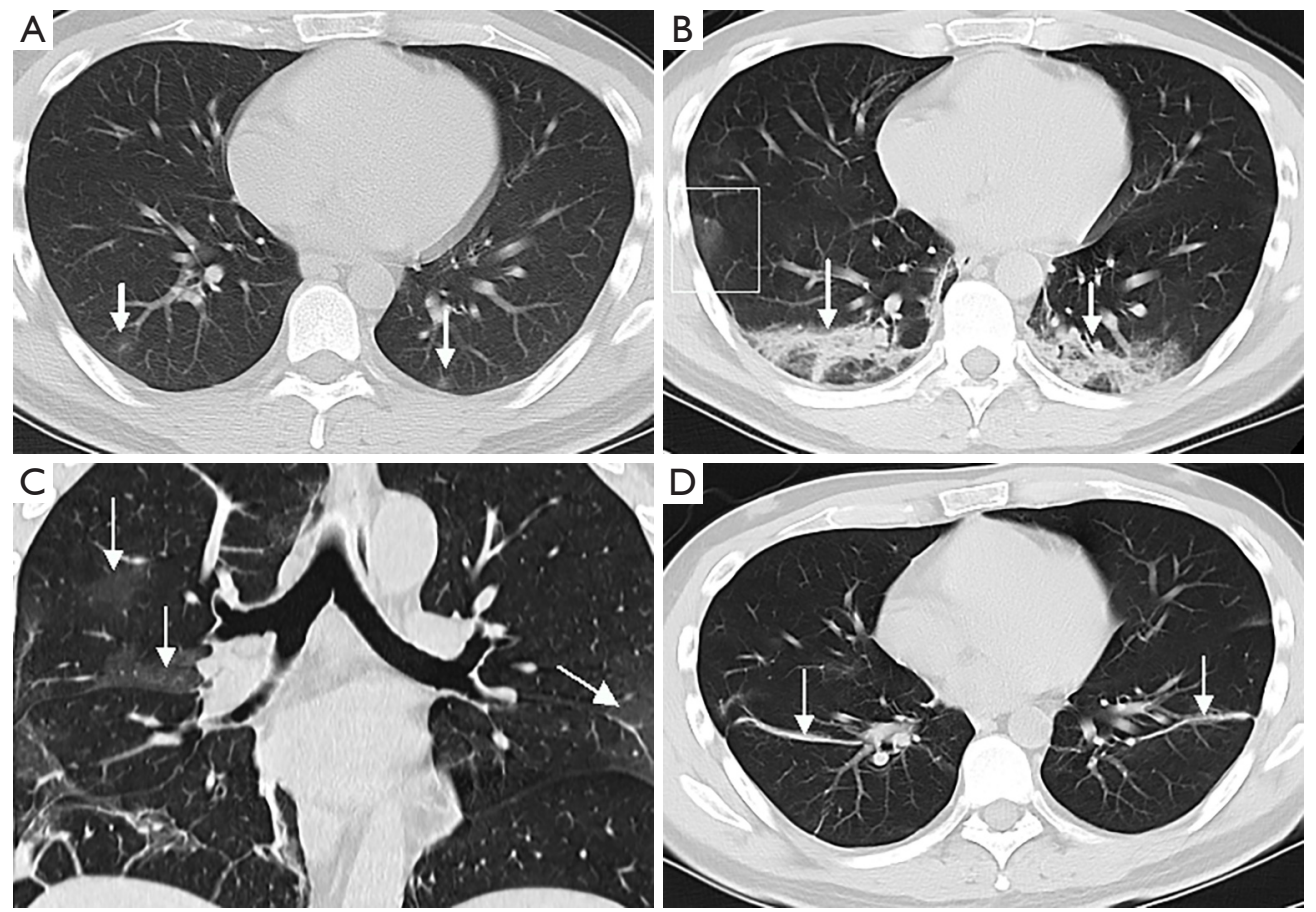

Figure 4 A series of CT images in a 31-year-old man with history of recent stay in Wuhan who presented with fever. (A) Axial CT image obtained from the day symptom just emerged shows subtle GGOs on subpleural area of bilateral lower lobes (arrows); (B) axial CT image obtained on day eight shows that GGOs developed partial consolidation (arrows) and new GGO displayed on right lobe (box); (C) reconstructed coronal CT image obtained on day 13 shows that GGO in right lower lobe developed irregular and fibrous pattern and GGOs extended to more lobes (arrows). The severity of lung injury was then categorized grade 2; (D) axial image obtained on day 23 shows lesions have been almost completely resolved with fibrous stripe left (arrows). This case was clinically classified to severe group, which consistent with CT grade 2. 

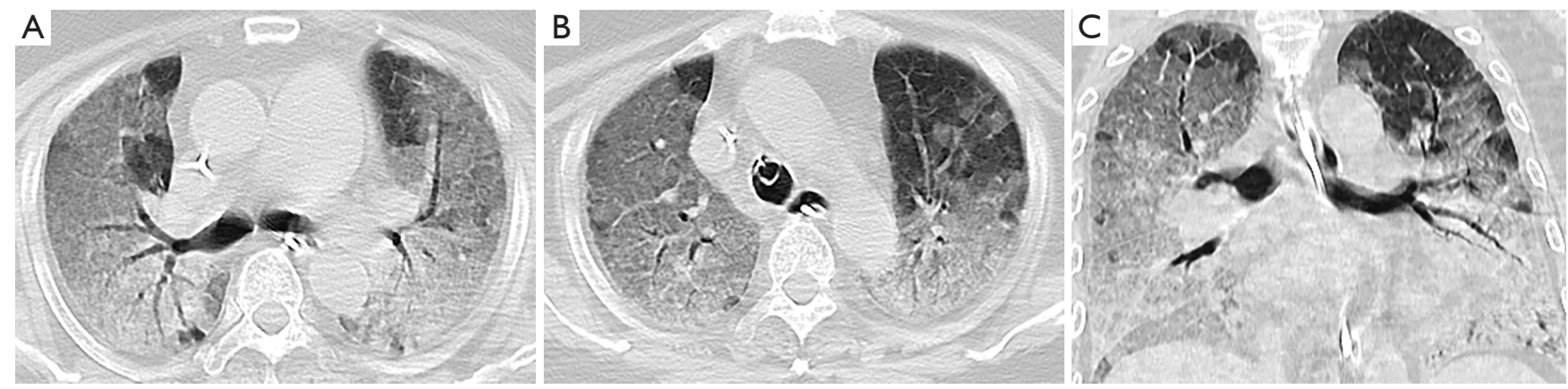

Figure 5 A 65-year-old female patient with unknown exposure history displayed fever and cough initially and progressed to dyspnea. (A,B) Axial CT images obtained 15 days after admission show diffuse opacity with "air bronchogram" sign; (C) coronal CT image shows that almost whole bilateral lung affected by the diffuse lesion. This patient was admitted to ICU and performed mechanical ventilation. The severity of lung injury on CT was grade 3 and consistent with clinical critical group.

Although the clinical course of patient remains to be fully characterized, early isolation and supportive treatments was recommended for the infected patients at early stage (18). Therefore, rapid disease recognition for suspected patients is crucial to speed up treatment and prompt isolation. As a COVID-19 designated medical center, our hospital has drafted a protocol at beginning of the outbreak using chest CT combined with RT-PCR to screen and detect suspected COVID-19 patients. A total of 29 patients from our clinic were definitively diagnosed as COVID-19 infection through this procedure (Figure 1). Importantly, three patients with negative results of the initial RT-PCR assay, has demonstrated GGOs or consolidations on the simultaneous chest CT scan. An analogous result has been firstly reported that out of 167 ultimately confirmed COVID-19 patients, five cases with initial negative RT-PCR, demonstrated typical chest CT findings of 2019-nCoV pneumonia (19), thus indicated the importance of chest CT examination in patient screening and early disease detection. Actually, a guideline for COVID-19 has recently been revised by China health authority and clinical diagnostic criteria have been adopted for Wuhan/Hubei province, the major endemic center of China (15). According to the localized criteria, diagnosis for COVID-19 would be clinically established if the suspected patient displayed typical radiological findings, despite of RT-PCR result. The implementation would allow public health for early surveillance, containment, and response to this communicable disease.

GGO and consolidation with peripheral distribution were the most common chest CT findings, which consistent with the previous study recently published (11-14). However, our patient sample was unique from those of other COVID-19 series in that 29 of our patients were introduced from the fever clinic, resulting in a relative brief interval between initial CT scan and onset of symptoms. One of these patients even has been performed CT examination just on the day symptom appeared, and peripheral subtle GGOs were displayed on CT images (Figure 4). Based on the finding, it would be concerned that GGO, usually appeared in lower lobes with striking peripheral distribution, may represent the very first radiographically visible manifestation of COVID-19.

Three patients out of current series have been particular noted, that displayed normal images on the initial chest CT scan, although ultimately confirmed COVID-19 infection. One of these developed GGOs in the bilateral lower lobes. While the other two remained entirely normal chest CT during whole hospitalization, thus radiologically graded 0 (Table 3). The negative imaging in confirmed infected patients suggests that chest CT scan lacks complete sensitivity and cannot alone fully exclude COVID-19.

In current series, only a 13-year-old boy was clinically classified to mild group (Table 3). This young patient presented with low fever for two days, without any other symptom displayed. While his parents have been confirmed COVID-19 and the RT-PCR assay was definitely revealed positive result. Moreover, no abnormal manifestation was found on both his initial and repeat CT scans. This sporadic case indicates COVID-19 may cause infection without lung injury involved. Although the CT grading is significantly consistent with clinical classification in our results (kappa $=0.638, \mathrm{P}<0.05$ ), it was noted that only 6 out of 17 clinical critical patients were graded 3 on CT imaging (Table 3). It was supposed partly because the severity of 


\section{Page 8 of 9}

lung changes visible on imaging is mostly related to the severity of the disease itself. While the critical group patients were more likely to have underlying comorbidities, including hypertension, diabetes and cardiovascular disease. Furthermore, compared with the whole series average age of 53.3 years (range, 13-96 years); the average age of critical group patients was 71.6 years (range, 37-96 years). Although recently some authors argue that the role of CT for COVID-19 patient's management remains poorly defined (20), CT grading was consistent with clinical classification indicate that chest CT series may help to categorize patients.

Our study had several limitations. All data in current series came from a single center, and then the proportion of critical cases and the mortality in our series might be inconsistent with previous reports from Wuhan, China $(6,8,9)$. For relative brief term of data collection, no enough follow-up CT and patients' outcome provided for our analysis.

In summary, we reported a screening system using chest CT combined RT-PCT assay to detect COVID-19 suspected patient and described the chest CT features of COVID-19 patient. Peripheral lung distributed GGO and consolidation, without subpleural sparing, are the most common manifestations on chest CT of COVID-19 infected patients. Abnormalities on chest CT can appear in a very early stage of COVID-19, thus help to early recognition and rapid diagnosis of this disease. Furthermore, we firstly report that the severity of lung involvement on chest CT is significantly consistent with clinical classification of COVID-19 patients. Then chest CT series may help to manage patients. Presently, worldwide public health measures are updating on a daily basis to manage this new outbreak, we hope our report could be of benefit not only to radiologist and physician but to the larger public health surveillance and response systems.

\section{Acknowledgments}

Funding: This work was supported in part by Zhejiang Provincial Natural Science Foundation (No. LY17H180001 to HZ) and by National Natural Science Foundation of China (No. 81701683 to FZ).

\section{Footnote}

Reporting Checklist: The authors have completed the STROBE reporting checklist. Available at http://dx.doi.
Zhou et al. CT benefit to COVID-19 recognition and classification

org/10.21037/atm-20-2119a

Data Sharing Statement: Available at http://dx.doi. org/10.21037/atm-20-2119a

Conflicts of Interest: All authors have completed the ICMJE uniform disclosure form (available at http://dx.doi. org/10.21037/atm-20-2119a). FZ serves as an unpaid section editor of Annals of Translational Medicine from Jan 2020 to Dec 2020. The other authors have no conflicts of interest to declare.

Ethical Statement: The authors are accountable for all aspects of the work in ensuring that questions related to the accuracy or integrity of any part of the work are appropriately investigated and resolved. The study protocol was approved by the Research Ethics Committee of the First Affiliated Hospital of Zhejiang University School of Medicine.

Open Access Statement: This is an Open Access article distributed in accordance with the Creative Commons Attribution-NonCommercial-NoDerivs 4.0 International License (CC BY-NC-ND 4.0), which permits the noncommercial replication and distribution of the article with the strict proviso that no changes or edits are made and the original work is properly cited (including links to both the formal publication through the relevant DOI and the license). See: https://creativecommons.org/licenses/by-nc-nd/4.0/.

\section{References}

1. National Health Commission of the People's Republic of China. Latest on the novel coronavirus outbreak. Available online: http://en.nhc.gov.cn/2020-01/29/c_76043.htm (accessed Jan 29, 2020).

2. Lu H, Stratton CW, Tang YW. Outbreak of pneumonia of unknown etiology in Wuhan, China: The mystery and the miracle. J Med Virol 2020;92:401-2.

3. Zhou P, Yang XL, Wang XG, et al. A pneumonia outbreak associated with a new coronavirus of probable bat origin. Nature 2020;579:270-3.

4. Zhu N, Zhang D, Wang W, et al. A Novel Coronavirus from Patients with Pneumonia in China, 2019. N Engl J Med 2020;382:727-33.

5. Tan W, Zhao X, Ma X, et al. A novel coronavirus genome identified in a cluster of pneumonia cases-Wuhan, China 2019-2020. Available online: http://weekly.chinacdc.cn/ 
en/article/id/a3907201-f64f-4154-a19e-4253b453d10c (accessed Jan 23, 2020).

6. Huang C, Wang Y, Li X, et al. Clinical features of patients infected with 2019 novel coronavirus in Wuhan, China. Lancet 2020;395:497-506.

7. WHO. Novel coronavirus (2019-nCoV). Situation report-14. Feb 3, 2020. Available online: https:// www.who.int/docs/default-source/coronaviruse/ situation-reports/20200203-sitrep-14-ncov. pdf?sfvrsn=f7347413_2 (accessed Feb 3, 2020).

8. Chen N, Zhou M, Dong X, et al. Epidemiological and clinical characteristics of 99 cases of 2019 novel coronavirus pneumonia in Wuhan, China: a descriptive study. Lancet 2020;395:507-13.

9. Wang D, Hu B, Hu C, et al. Clinical Characteristics of 138 Hospitalized Patients With 2019 Novel CoronavirusInfected Pneumonia in Wuhan, China. JAMA 2020;323:1061-9.

10. Liu K, Fang YY, Deng Y, et al. Clinical characteristics of novel coronavirus cases in tertiary hospitals in Hubei Province. Chin Med J (Engl) 2020;133:1025-31.

11. Chung M, Bernheim A, Mei X, et al. CT Imaging Features of 2019 Novel Coronavirus (2019-nCoV). Radiology 2020;295:202-7.

12. Pan Y, Guan H, Zhou S, et al. Initial CT findings and temporal changes in patients with the novel coronavirus pneumonia (2019-nCoV): a study of 63 patients in Wuhan, China. Eur Radiol 2020;30:3306-9.

Cite this article as: Zhou $\mathrm{H}, \mathrm{Xu} \mathrm{K}$, Shen $\mathrm{Y}$, Fang Q, Chen F, Sheng J, Zhao F, Lou H. Coronavirus disease 2019 (COVID-19): chest CT characteristics benefit to early disease recognition and patient classification-a single center experience. Ann Transl Med 2020;8(11):679. doi: 10.21037/ atm-20-2119a
13. Fang Y, Zhang H, Xu Y, et al. CT Manifestations of Two Cases of 2019 Novel Coronavirus (2019-nCoV) Pneumonia. Radiology 2020;295:208-9.

14. Lin X, Gong Z, Xiao Z, et al. Novel Coronavirus Pneumonia Outbreak in 2019: Computed Tomographic Findings in Two Cases. Korean J Radiol 2020;21:365-8.

15. China National Health Commission. Diagnosis and treatment of pneumonitis caused by new coronavirus (trial version 5). Beijing: China National Health Commission, 2020. Available online: http://www.nhc.gov.cn/yzygj/s7653 p/202002/3b09b894ac9b4204a79db5b8912d4440.shtml

16. Kanne JP. Chest CT Findings in 2019 Novel Coronavirus (2019-nCoV) Infections from Wuhan, China: Key Points for the Radiologist. Radiology 2020;295:16-7.

17. Hansell DM, Bankier AA, MacMahon H, et al. Fleischner Society: glossary of terms for thoracic imaging. Radiology 2008;246:697-722.

18. Jin $\mathrm{YH}$, Cai L, Cheng ZS, et al. A rapid advice guideline for the diagnosis and treatment of 2019 novel coronavirus (2019-nCoV) infected pneumonia (standard version). Mil Med Res 2020;7:4.

19. Xie X, Zhong Z, Zhao W, et al. Chest CT for Typical 2019-nCoV Pneumonia: Relationship to Negative RTPCR Testing. Radiology 2020. [Epub ahead of print]

20. Wang YXJ, Liu WH, Yang M, et al. The role of CT for Covid-19 patient's management remains poorly defined. Ann Transl Med 2020;8:145. 